\title{
A Distributed Trust Diffusion Protocol for Ad Hoc Networks
}

\author{
Michel Morvan ${ }^{1,2,4}$ and Sylvain Sené (3,4* $^{2}$ \\ ${ }^{1}$ Université de Lyon, LIP, \\ 46, allée d'Italie, 69364 Lyon cedex 07, France \\ 2 École des hautes études en sciences sociales and Santa Fe Institute \\ ${ }^{3}$ UJF-Grenoble, TIMC-IMAG, \\ Faculté de médecine, 38706 La Tronche cedex, France \\ ${ }^{4}$ IXXI, Institut rhône-alpin des systèmes complexes \\ 5, rue du Vercors, 69007 Lyon, France
}

\begin{abstract}
In this paper, we propose and evaluate a distributed protocol to manage trust diffusion in ad hoc networks. In this protocol, each node $i$ maintains a "trust value" about an other node $j$ which is computed both as a result of the exchanges with node $j$ itself and as a function of the opinion that other nodes have about $j$. These two aspects are respectively weighted by a trust index that measures the trust quality the node has in its own experiences and by a trust index representing the trust the node has in the opinions of the other nodes. Simulations have been realized to validate the robustness of this protocol against three kinds of attacks: simple coalitions, Trojan attacks and detonator attacks.

Keywords: trust diffusion, ad hoc network, interaction graph.
\end{abstract}

\section{Introduction and preliminaries}

Ad hoc networks can be defined as collections of mobile nodes, distributed and independent, being able to communicate by radio transmission and to self-organise. They constitute networks with unstable infrastructure. Since

${ }^{*}$ Corresponding author. Email address: Sylvain.Sene@ens-lyon.fr 
the neighbourhood of every node changes over time, it is important to develop protocols that will help each node to identify reliably other nodes with which it can interact safely.

Let us present two definitions of the concept of trust given by the Webster's classic 1913: "Firm reliance on the integrity, ability, or character of a person or thing" and "Certainty based on past experience". Trust is therefore a natural notion acquired and systematically used by anyone to decide if an exchange with somebody else is conceivable or not. Let us remark that the trust we can have in somebody usually depends on the personal knowledges we have about the person and also on his/her reputation according to other people we have already met. This is usually the way that trust diffuses in human groups. For example, a researcher can trust the scientific opinion of a colleague while not trusting the opinion of the same colleague about the work of other scientists. The idea of this paper is to transfer this human trust diffusion protocol to ad hoc networks. Literature about trust management in P2P systems BB04, KSGM03 and Web Services RZ01 presents some solutions using both personal and external opinions. However, these protocols do not offer protection against attacks made by a set of nodes working in coalition, for example diffusing wrong positive opinions about a dishonest node (called Trojan attack further in the paper). That is why we propose to study in this paper a management policy that also measures the trust quality by introducing a new level of trust that adds weights both on a node own experiences and on external knowledges this node has received from the others.

Let us call efficient a protocol that leads to prevent bad interaction and reliable a protocol that aims at favouring good interactions. This work consists in creating, studying and validating by simulations a new efficient distributed trust diffusion protocol for ad hoc networks using the double level of trust described above.

Section 2 presents and explains the trust diffusion protocol. Some results obtained on this protocol are exposed in Section 3. In particular, this section shows how the protocol can resist to three different types of attacks. Section 1 gives perspectives of this work.

\section{Trust diffusion protocol}

Because of the constraints inherent to ad hoc networks, the proposed model has to be based on three fundamental concepts: information decentralisation, diffusion and management of trust histories. 
In this section, we first present the different attributes used by the protocol in order to have the requested features to adapt on ad hoc networks. Then, we expose the protocol dynamics.

\subsection{Protocol attributes}

In order to respect the constraints induced by ad hoc networks, all nodes have to store their own knowledges about the others. This subsection introduces the different attributes that compose the notion of knowledge used in the proposed protocol and that a node $i$ has about a node $j$ at time $t$.

- Trust mark, denoted by $t m_{i, j}(t) \in[0,1]$.

Node $i$ stores a trust mark valued in $[0,1]$ about node $j$ with which it has already interacted. This trust mark is used to know if an interaction has a good chance to be benefic. It is updated as a function of two major information: the own experience of node $i$ and the external knowledges node $i$ has obtained from other nodes during past interactions. It is initialized to $\frac{1}{2}$.

- Trust index in trust mark, denoted by $i t m_{i, j}(t) \in[0,1]$.

Node $i$ stores a trust index about its trust mark about node $j$. This trust index gives an indication about the reliability of the trust mark it has about node $j$. It is initialized to $\frac{1}{2}$.

- List of external trust knowledges, denoted by $e_{i, j}(t)$ with $e t_{i, j}(t)[k] \in$ $[0,1]$ and $k \in[0, n]$.

Node $i$ stores about node $j$ a list of external trust marks. Such a list contains the trust marks owned by $j$ about other nodes. This list has been transmitted to node $i$ during the last interaction between $i$ and $j$ (during this interaction, $i$ has symmetrically transmitted its trust marks to $j$ ). This list is initialized to $\emptyset$. Moreover, when nodes $i$ and $j$ symmetrically transmit to each other their trust marks, they also transmit their trust indices in these trust marks. These lists of trust indices are called lists of external trust indices in trust knowledges. Such a list owned by node $i$ about node $j$ is denoted at time $t$ by $i e l_{i, j}(t)$ with $i e l_{i, j}(t)[k] \in[0,1]$ and $k \in[0, n]$ and is initialized to $\emptyset$.

- Trust index in list of external trust knowledges, denoted by $i e t_{i, j}(t) \in$ $[0,1]$.

Node $i$ stores a trust index about each list of external knowledges it has received from other nodes. Such trust indices give an indication about the reliability of these lists and are initialized to $\frac{1}{2}$. 
- List of past interactions marks, denoted by $p i l_{i, j}(t)$ with $p i l_{i, j}(t)[k] \in$ $\{0,1\}$ and $k \in[0, \theta]$.

Node $i$ stores about node $j$ a list of marks encoding the results of its $\theta$ (where $\theta$ is bounded by $\theta_{\max }$ to avoid side effects) last interactions with the node. If the interaction was a success (the transmitted data was the expected data), the mark is 1 , and it is 0 otherwise. The marks included in this list correspond to the own experience of node $i$ about node $j$ and are used to compute trust mark for future interactions. It is initialized to $\emptyset$.

We will call positive (resp. negative) an attribute (valued in $[0,1]$ ) that is greater or equal to (resp. less than) $\frac{1}{2}$.

\subsection{Protocol dynamics}

We describe in this subsection the updating methods of the protocol attributes described above. First, let us note that the list of external trust knowledges (et) and the list of external trust indices in trust knowledges $(\mathrm{iel})$ are simply transmitted through the network by nodes at each interaction. Then, the update of the list of past interactions is done as follows: when node $i$ asks node $j$ for data transmission, if the list of past interactions of $i$ is not full, the mark affected to this new interaction is placed at the end of the list; otherwise, the first mark in the list is removed and the new mark is placed at the end. Now, let us focus on other attributes that need more complicated updating functions.

\section{- Trust marks}

When node $i$ wishes to interact with node $j$ at time $t$, it has not only to rely on its own knowledges but also on the external knowledges about node $j$ it has already received from the other nodes. Thus, to update its trust mark about node $j$, node $i$ needs to evaluate two different data. First, we define the personal knowledges PK as the simple average of the results of past interactions node $i$ had with node $j$. So:

$$
\mathrm{PK}_{\mathrm{i}, \mathrm{j}}^{\mathrm{t}+1}=\frac{\sum_{\mathrm{k}=1}^{\theta} \mathrm{pil}_{\mathrm{i}, \mathrm{j}}(\mathrm{t})[\mathrm{k}]}{\theta}
$$

Now, let us also define the external knowledges. Let $\mathrm{S}^{*}$ be the set of nodes $k$ such as $\left\{\mathrm{k} \neq \mathrm{i}, \mathrm{j}\right.$, $\operatorname{iet}_{\mathrm{i}, \mathrm{k}}(\mathrm{t})>\frac{1}{2}$, et $\left.\mathrm{t}_{\mathrm{i}, \mathrm{k}}(\mathrm{t})[\mathrm{j}]<\frac{1}{2}, \operatorname{iel}_{\mathrm{i}, \mathrm{k}}(\mathrm{t})[\mathrm{j}]>\frac{1}{2}\right\}$. At time $t+1$, the external knowledges EK that node $i$ has about node $j$ are computed as: 


$$
\mathrm{EK}_{\mathrm{i}, \mathrm{j}}^{\mathrm{t}+1}=\frac{1}{\left|\mathrm{~S}^{*}\right|} \sum_{\substack{\mathrm{k}=1 \\ \mathrm{~S}^{*}}}^{\mathrm{n}} \mathrm{et}_{\mathrm{i}, \mathrm{k}}(\mathrm{t})[\mathrm{j}] .
$$

The choice made here is to average only the bad opinions other nodes have about node $j$. Of course, we will restrict to nodes in which we trust the opinion and who themselves trust their own opinion about node $j$. Note that this choice leads the protocol to be efficient rather than reliable.

Now, we update the trust mark by simply taking the weighted sum of these two knowledge values:

$$
\operatorname{tm}_{\mathrm{i}, \mathrm{j}}(\mathrm{t}+1)=\frac{\varepsilon \cdot\left[\theta \times \mathrm{PK}_{\mathrm{i}, \mathrm{j}}^{\mathrm{t}+1}\right]+\overbrace{\zeta \cdot\left[\theta_{\max } \times E K_{i, j}^{t+1}\right]}^{(\mathrm{E})}}{\varepsilon \cdot \theta+\underbrace{+\theta_{\max }}_{\text {iff }(\mathrm{E}) \neq 0}}
$$

where $\varepsilon$ and $\zeta$ are two parameters managing the weight of personal and external knowledges. So, the more $\zeta$ has a high value compared to $\varepsilon$, the more the diffusion will be fast. However, $\varepsilon$ and $\zeta$ have to be well balanced to prevent the fact a node is easily disturbed if it has a majority of dishonest neighbours. Let us note that, if these two parameters are equal, external knowledges weight is greater than personal knowledges weight when the $\theta_{\max }$ first interactions have not already been executed, because $\theta_{\max }$ is the upper bound of $\theta$. It seems to be reasonable to think that, when a node has few personal knowledges about an other with which it wants to interact, external knowledges are more important in its decision.

This trust marks evolution method shows that this protocol can not be less efficient than another one which does not implement trust diffusion. Indeed, if an interaction between two nodes $i$ and $j$ is possible, when $i$ updates its trust mark about $j$, it can not obtain a higher new mark than one obtained only with its past interactions with $j$ since it only uses negative external knowledges.

\section{- Trust indices in personal trust marks}

The updating method of the trust mark index (when $i$ asks $j$ for an interaction at time $t+1)$ is the following. After having computed $E\left(p i l_{i, j}(t)\right)$ and $\sigma\left(p i l_{i, j}(t)\right)$ where $E$ and $\sigma$ refer respectively to the arithmetic average and the standard deviation, the new value of the trust mark index is obtained by a threshold algorithm. So, we fix a threshold named $\omega_{i t m}$ and we verify 
if the new trust mark $t m_{i, j}(t+1)$ is in

$$
] \mathrm{E}\left(\mathrm{pil}_{\mathrm{i}, \mathrm{j}}(\mathrm{t})\right)-\omega_{\mathrm{itm}} ; \mathrm{E}\left(\mathrm{pil}_{\mathrm{i}, \mathrm{j}}(\mathrm{t})\right)+\omega_{\mathrm{itm}}[
$$

The index itm grows if $\alpha$ is in this range of values and decreases otherwise. In each case, updates are proportional to $\sigma\left(p i l_{i, j}(t)\right)$; however, in order to go one step further in the "efficiency" direction, we have chosen the decreasing function to be faster than the increasing one.

\section{- Trust indices in external trust marks}

Node $i$ will use the newly calculated trust mark on node $j$ to adjust the trust index it has about the external trust knowledges stored for each other node. In other words, for each other node $k$, node $i$ compares its new trust mark about $j$ to the opinion $k$ has about $j$. The more different (resp. close) it is, the more node $i$ decreases (resp. increases) its trust index on the list of external knowledges given by $k$. A threshold algorithm (where the threshold is named $\omega_{i e t}$ ) is used here too. When $i$ interacts with $j$, we consider the belonging of each $e t_{i, k}(t)[j]$ to the following range of values:

$$
] \operatorname{tm}_{i, j}(t+1)-\omega_{\text {iet }} ; \operatorname{tm}_{i, j}(t+1)+\omega_{\text {iet }}[
$$

If $e t_{i, k}(t)[j]$ is in this range of values, the index $i e t_{i, k}(t)$ grows, otherwise it decreases. There also, the decreasing goes faster than the growth.

\subsection{Decision to interact}

At time $t+1$, if node $i$ wishes to interact with node $j$, it verifies its trust mark about node $j$ and decides to realize the interaction only if this trust mark is positive.

\section{Simulations}

In this section, we present the simulations that we have done on the trust diffusion protocol presented above (called Trudi) and compare the results of the latter to the so-called $\beta$-protocol in which nodes do not transmit their knowledges about other nodes. We are going to evaluate the efficiency of the protocols by measuring three parameters: (i) the number of honest nodes succeeding in identifying the dishonest nodes; (ii) the total number of bad interactions (transmitted data are not the expected ones) that the dishonest nodes succeeded to do; (iii) in some cases, we also measure the number of interactions needed for the honest nodes to identify the dishonest ones. 
Let us precise the meaning of honest and dishonest we will use in the following. We will call action-dishonest (resp. action-honest) a node that wishes to give to every other unexpected data (resp. expected data) and opinion-dishonest (resp. opinion-honest) a node that wishes to give wrong opinions (resp. correct opinions) about some nodes to many others. A node is called dishonest if it is either action-dishonest or opinion-dishonest and totally-dishonest if it is both action-dishonest and opinion-dishonest.

Moreover, a trust protocol is called coherent if, when a little proportion of the system actors is action-dishonest, the honest ones succeed in the detection of those latter.

We will start by describing the simulation protocol used in the study before exposing some of the obtained results.

\subsection{Simulation protocol}

In order to run simulations not to far from real interactions in ad hoc networks, we are going to make the hypothesis that if we observe the set of interactions in the network after a sufficient amount of time, we will observe a network close either to a social network or to a complete network (where each node has interacted at least once with any other one). A social network is the personal or professional set of relationships between individuals. It has been shown [AB02, New03 that these networks very often present the following characteristics: their degree distribution follows a power law (the probability that a node has a degree $k$ is a decreasing power of $k$ ); they present a clustering coefficient (the probability for two nodes having a common neighbour to be connected) significantly higher than for classical random networks [SW98]. Of course, there is no evidence that these social networks precisely corresponds to the reality, but it makes sense to suppose that interactions in ad hoc networks will share some features with interactions in social networks.

In order to run simulations, that will lead at the end to such interaction networks, we determine the interaction graph a priori and we then realize the interactions on this graph. Thanks to this method, we control the final shape of the interactions superposition. Because of the above hypothesis, the chosen graphs are either power law degree distribution graphs with high clustering coefficient (such a network will be called "social network" in the following) or complete graphs. 


\subsection{Simulations results}

The results presented in this subsection come from simulations of 1000000 interactions on 100 nodes networks.

In the first simulation, there is only one action-dishonest node which sends bad data with probability $\frac{1}{5}$ and no opinion-dishonest node. In the social network, this node is given the highest degree. We measure the average and maximum number of interactions realized by the other nodes with this action-dishonest one. In Table 1, which presents the results of this simulation, we observe that Trudi is significantly better than the $\beta$-protocol. Indeed, the perception of dishonesty is about 45 times faster with Trudi than with the $\beta$-protocol. This result can be explained by the nature of trust diffusion in Trudi. Only negative marks and opinions are used to realize the updates of the protocol attributes. Therefore, as the weight of external knowledges is always more important than this of personal knowledges, honest nodes perceive quasi-directly the dishonesty.

Another simulation has been run by placing the dishonest node on the lowest degree node. In this case, there is no real difference between the two protocols because of the too small connectivity of the node. Thus, the interest of the protocol depends on the probability dishonest nodes have to give bad interactions and on the place of dishonesty in the network, an effect that we will call topology dependence.

Table 1: Comparison of Trudi and the $\beta$-protocol after a simple attack.

\begin{tabular}{|c|c|c|}
\hline & Trudi & $\beta$-protocol \\
\hline \hline Avg ; Max & $3.38 ; 10$ & $153.04 ; \sim 420$ \\
\hline
\end{tabular}

Let us now assume that some nodes make a coalition in order to attack the network, i.e. to use their knowledge about the protocol to break its efficiency or reliability. We are going to present in the following some results describing the effects that such an attack can have, with and without the Trudi protocol.

\section{- Simple coalition attacks}

A simple coalition attack manages a group of nodes in which the members are honest with each other but totally-dishonest with nodes that are not a part of the group, i.e. they protect themselves by giving positive opinions about the group members outside the group.

We have run simulations of this type of attack on complete networks. In each simulation, we have increased the size of the group of dishonest nodes 
(which send bad data with probability $\frac{1}{5}$ ) to focus on the minimal size this group must have to destabilise the network, i.e. at least one honest node does not succeed in perceiving all the dishonest ones.

Table 2: Comparison of Trudi and the $\beta$-protocol after coalition attacks.

\begin{tabular}{|c|c|c|}
\hline Number of dishonest nodes in the group & Trudi & $\beta$-protocol \\
\hline \hline 40 & $\sqrt{ }$ & $\times$ \\
\hline 50 & $\sqrt{ }$ & $\times$ \\
\hline 60 & $\sqrt{ }$ & $\times$ \\
\hline 70 & $\times$ & $\times$ \\
\hline 80 & $\times$ & $\times$ \\
\hline
\end{tabular}

Table 2 shows that Trudi is extremely robust against simple coalitions contrary to the $\beta$-protocol. With Trudi, the system can actually be destabilised only if the rate of good interactions is much less than the rate of bad interactions provided by dishonest nodes. Let us illustrate these results on a simple example which models a complete network where every dishonest node has a probability of $\frac{1}{5}$ to provide a bad interaction. At each interaction, an edge has a probability of $\frac{1}{9900}$ to be chosen (the interactions from $i$ to $j$ and from $j$ to $i$ are differentiated). So, when only thirty nodes are honest, the probability for two honest nodes to interact is $\frac{30 \times 29}{9900} \approx 0.0878$. When forty nodes are honest, this probability is $\frac{40 \times 39}{9900} \approx 0.1575$. So, in the second case, the system is not destabilised because the rate of good knowledges diffusion is close to the rate of bad interactions provided by dishonest nodes. This explanation has been verified by other simulations.

Moreover, note that the results presented in Table 2 are also valid for social networks despite the topology-dependence.

Let us now considerer more sophisticated attacks.

\section{- Trojan attacks}

A Trojan attack (by analogy to the mythical war of Troy) manages a group of nodes where one of them (Ulysses) is honest inside the group and totally-dishonest outside the group. Ulysses is protected by the other members of the group, i.e. they are action-honest but they always provide positive opinion to the honest nodes about Ulysses.

For this attack, two simulations have been executed (illustrating protocols robustness both on complete and social networks) to see the number of bad interactions $(n b i)$ realized by Ulysses (which sends bad data with 
probability $\frac{1}{5}$ ) and the maximum number of interactions ( $\left.m n i\right)$ executed by a honest node with Ulysses before perceiving its dishonesty.

Table 3: Comparison of Trudi and the $\beta$-protocol after Trojan attacks.

\begin{tabular}{|c|c|c|}
\hline $\begin{array}{c}\text { Type of network and } \\
\text { Ulysses error probability }\end{array}$ & Trudi & $\beta$-protocol \\
\hline \hline Complete network $-20 \%$ & $\mathrm{nbi}=52 ; \mathrm{mni}=8$ & $\mathrm{nbi}=1233 ; \mathrm{mni}=130$ \\
\hline Social network $-20 \%$ & $\mathrm{nbi}=14 ; \mathrm{mni}=25$ & $\mathrm{nbi}=344 ; \mathrm{mni} \sim 900$ \\
\hline
\end{tabular}

In the second simulation, Ulysses is the highest degree node and is protected by 9 high degree nodes. Table 3 validates the robustness of Trudi against Trojan attacks on complete networks as well as on social networks. However, other simulations where the dishonest group takes place on nodes of lowest degrees have shown that Trudi efficiency is not really significantly more interesting than one of the $\beta$-protocol. These results confirm that topology dependence is the main factor of the interest of the protocol in social networks.

\section{- Detonator attacks}

A detonator attack is a Trojan attack where the dishonesty of Ulysses is started only after a determined time which is called the detonation time.

Our objective is to determine how many bad interactions (nbi) are provided by Ulysses, after its detonation, to be recognised as a mistrustable node and how many honest nodes $(n h n)$ succeed in perceiving the dishonesty of Ulysses.

Table 4: Comparison of Trudi and the $\beta$-protocol after detonator attacks.

\begin{tabular}{|c|c|c|}
\hline $\begin{array}{c}\text { Number of interaction } \\
\text { after the detonation }\end{array}$ & Trudi & $\beta$-protocol \\
\hline \hline $\begin{array}{c}\text { Complete network } \\
500000\end{array}$ & $\mathrm{nbi}=836 ; \mathrm{nhn}=19$ & $\mathrm{nbi}=837 ; \mathrm{nhn}=12$ \\
\hline $\begin{array}{c}\text { Complete network } \\
900000\end{array}$ & $\mathrm{nbi}=1051 ; \mathrm{nhn}=75$ & $\mathrm{nbi}=1411 ; \mathrm{nhn}=27$ \\
\hline \hline $\begin{array}{c}\text { Social network } \\
900000\end{array}$ & $\mathrm{nbi}=419 ; \mathrm{nhn}=15$ & $\mathrm{nbi}=657 ; \mathrm{nhn}=15$ \\
\hline
\end{tabular}


Two simulations have been run to show the robustness of the two protocols against detonator attacks in complete networks where Ulysses sends bad data with probability $\frac{1}{5}$ and is protected by 9 other nodes.

The first (resp. the second) simulation illustrates this type of attack where Ulysses becomes dishonest after the 500000th (resp. 100000th) interaction. Table 4 shows that a too little number of interactions executed after the detonation of Ulysses node prevents many honest ones to perceive its dishonesty. Indeed, as the interactions are chosen randomly in the $\dot{n(n-1)}$ possible, in a 100 nodes system, $9900(\approx 10000)$ different interactions exist. Consequently, an edge between a given node and Ulysses is potentially chosen fifty times in the first simulation. It is not sufficient for the given node to detect the dishonesty of Ulysses because of two reasons. Firstly, the given node has already a positive opinion about Ulysses and, secondly, the latter has a too small probability to provide bad interactions $\left(p=\frac{1}{5}\right)$. The second line of Table 4 validates this hypothesis. If we focus on the number of bad interactions, we can conclude our protocol is interesting because it provides a mean of 11.68 of bad interactions to each honest node.

Then, another simulation with the same features than the second one has been run to show the robustness of the two protocols against these attacks in social networks. Ulysses is here the node with the highest degree (with 15 honest neighbours). The last line of Table 4 shows that, with the two protocols, all Ulysses neighbours succeed in perceiving its dishonnesty. However, with Trudi, Ulysses node gives less bad data than with the $\beta$ protocol. Moreover, if we focus on the total number of interactions realized by the 15 neighbours of Ulysses after the detonation time, we see that this number is approximately three times more important with the $\beta$-protocol than with Trudi.

\section{Conclusion and perspectives}

The results presented in this paper show that such a trust diffusion protocol could have interesting efficiency properties. Besides, it could apply not only in ad hoc networks but also in other networks. It could be of interest in Internet and P2P systems. For example, it could be useful to measure trust users give to web sites and could broadcast sites dishonesty. However, to obtain such protocols, improvements have to be done.

In particular, future research should focus on the reduction of the size of the transmitted messages because the protocols "lightness" is a primordial point to avoid the network surcharge. So, we should try to find a good 
balance between reducing the transmitted messages size and the interest of the diffusion principle. Different solutions could be imagined. For example, since the goal of the protocol is to ensure efficiency, we could only diffuse the information about bad behaving nodes. Another solution would be to diffuse only a proportion (function of the network size) of these negative knowledges. Such hypotheses should be tested via new simulations processes to be validated.

\section{References}

[AB02] Reka Albert and Albert-László Barabási. Statistical Mechanics of Complex Networks. Reviews of Modern Physics, 74(1):47-97, 2002 .

[BB04] Sonja Buchegger and Jean-Yves Le Boudec. A Robust Reputation System for P2P and Mobile Ad-hoc Networks. P2P and Mobile Ad-hoc Networks, Second Workshop on the Economics of Peer-to-Peer Systems, 2004.

[KSGM03] Sepandar D. Kamvar, Mario T. Schlosser, and Hector GarciaMolina. The EigenTrust Algorithm for Reputation Management in $\mathrm{P} 2 \mathrm{P}$ networks. The Twelfth International World Wide Web Conference, 2003.

[New03] Mark E. J. Newman. The Structure and Function of Complex Networks. Reviews of Society for Industrial and Applied Mathematics, 45(2):167-256, 2003.

[RZ01] Paul Resnick and Richard Zeckhauser. Trust among Strangers in Internet Transactions: Empirical Analysis of Ebay's Reputation System. Working Paper for the NBER Workshop on Empirical Studies of Electronic Commerce, 2001.

[SW98] Steven H. Strogatz and Duncan J. Watts. Collective Dynamics of 'Small-World' Networks. Nature, 393:440-442, 1998. 